\title{
Article
}

\section{A study of the power-cosine copula}

\section{Christophe Chesneau}

Université de Caen Normandie, LMNO, Campus II, Science 3, 14032, Caen, France; christophe.chesneau@gmail.com

Academic Editor: Qaisar Mehmood

Received: 9 June 2021; Accepted: 15 June 2021; Published: 29 June 2021.

Abstract: Copulas played a key role in numerous areas of statistics over the last few decades. In this paper, we offer a new kind of trigonometric bivariate copula based on power and cosine functions. We present it via analytical and graphical approaches. We show that it may be used to create a new bivariate normal distribution with interesting shapes. Subsequently, the simplest version of the suggested copula is highlighted. We discuss some of its relationships with the Farlie-Gumbel-Morgensten and simple polynomial-sine copulas, establish that it is a member of a well-known semi-parametric family of copulas, investigate its dependence domains, and show that it has no tail dependence.

Keywords: Copulas; Farlie-Gumbel-Morgenstern copula; Polynomial-sine copula; Normal distribution; Tail dependence.

MSC: $62 \mathrm{H} 99$.

\section{Introduction}

$\mathbf{M}$

odeling random multivariate events is a major topic in many scientific disciplines and creating multivariate distributions that can accurately model the variables at play is difficult. The concept of copula is a good tool for dealing with this issue. Basically, a copula is defined as a multivariate cumulative distribution function $(\mathrm{CDF})$ where each of the marginal CDFs is the CDF of the unit uniform distribution over the unit interval $(0,1)$. The first version of copula was published in 1959 [1], and the concept has received a great deal of attention since then, becoming the subject of numerous scientific studies. A wide range of applications in applied sciences can be found in [2-7], to name a few.

A bivariate copula, by definition, is a bivariate function $C(u, v),(u, v) \in[0,1]^{2}$ satisfying, for any $(u, v) \in$ $[0,1]^{2}, C(u, 0)=C(0, v)=0, C(u, 1)=u, C(1, v)=v, C(1,1)=1$, and, for any $\left(u_{1}, u_{2}, v_{1}, v_{2}\right) \in[0,1]^{4}$, such that $u_{1} \leq u_{2}$ and $v_{1} \leq v_{2}, C\left(u_{2}, v_{2}\right)-C\left(u_{2}, v_{1}\right)-C\left(u_{1}, v_{2}\right)+C\left(u_{1}, v_{1}\right) \geq 0$. It is demonstrated in [8] that this last condition is equivalent to $\partial^{2} C(u, v) / \partial u \partial v \geq 0$. The major result on the concept of copula is the Sklar theorem established in [1]. It ensures that, if $F(x, y)$ is a joint cumulative distribution function (CDF) with marginal CDFs given by $F_{X}(x)$ and $F_{Y}(y)$, respectively, then there exists a copula $C(u, v)$ such that $F(x, y)=C\left(F_{X}(x), F_{Y}(y)\right)$. The marginal distributions are thus isolated from the bivariate distribution's dependence structure.

A number of copulas have been presented over time. The Ali-Mikhail-Haq copula, Joe copula, Clayton copula, Frank copula, Gumbel-Hougaard copula, Farlie-Gumbel-Morgensten (FGM) copula, normal-type copulas, cubic-type copulas, simple polynomial-sine (SPS) copula and Cuadras-Augé copula are some examples. For the purposes of this paper, let us briefly present the FGM and SPS copulas. For $\lambda \in[-1,1]$, the FGM copula is defined by

$$
C_{\circ}(u, v)=u v[1+\lambda(1-u)(1-v)], \quad(u, v) \in[0,1]^{2} .
$$

The importance of the FGM copula is emphasized in [9-14], to name a few. On the other hand, among the rare trigonometric copulas proposed, for $\lambda \in[-1,1]$, the SPS copula is specified by

$$
C_{\triangle}(u, v)=u v+\lambda \frac{1}{\pi^{2}} \sin (\pi u) \sin (\pi v), \quad(u, v) \in[0,1]^{2} .
$$


It is known to have deep relationships with the FGM copula, as well as wider dependency domains to those associated to the FGM copula. We refer to $[15,16]$ for more details on the SPS copula. Other trigonometric bivariate copulas exist, including the more sophisticated ones described in $[17,18]$. In this paper, we introduce a new trigonometric copula based on power and cosine functions. The lack of simple trigonometric copula in the literature is one of the motivations of this study, opening some new horizons for bivariate modelling. First, we study its in-depth properties. Then, we use it to construct a new bivariate normal distribution with flexible bell shapes. The remaining part is devoted to the simplest form of the proposed copula. We focus on its properties, proving some ordering results involving the FGM and SPS copulas, highlighting the link with a more general well-identified family of copulas, and investigating the related dependence domain and tail dependence. All the elements are provided for future use of the new copula in a more applied setting.

The rest of the paper is conducted as follows: Section 2 defines the new copula, as well as its major features and direct applications. The simplest version of this copula is emphasized in Section 3. Section 4 summarizes the research findings.

\section{Power-cosine copula}

\subsection{First approach}

A new bivariate copula with power and cosine functions is proposed in the following statement:

Proposition 1. Let $\alpha, \beta>0$, and $\lambda \in\left[-c^{-1}, c^{-1}\right]$, where $c=\max (\alpha \pi / 2,1) \max (\beta \pi / 2,1)$. Then the following bivariate function is a copula:

$$
C_{\star}(u, v)=u v\left[1+\lambda \cos \left(\frac{\pi}{2} u^{\alpha}\right) \cos \left(\frac{\pi}{2} v^{\beta}\right)\right], \quad(u, v) \in[0,1]^{2} .
$$

Proof. We need to prove that the conditions that define a copula are fulfilled. We clearly have $C_{\star}(u, 0)=$ $C_{\star}(0, v)=0$, and, since $\cos (\pi / 2)=0$, it is clear that $C_{\star}(u, 1)=u, C_{\star}(1, v)=v$, and $C_{\star}(1,1)=1$. On the other hand, $C_{\star}(u, v)$ is twice differentiable with, after some developments,

$$
\frac{\partial^{2} C_{\star}(u, v)}{\partial u \partial v}=1+\lambda\left[\alpha \frac{\pi}{2} u^{\alpha} \sin \left(\frac{\pi}{2} u^{\alpha}\right)-\cos \left(\frac{\pi}{2} u^{\alpha}\right)\right]\left[\beta \frac{\pi}{2} v^{\beta} \sin \left(\frac{\pi}{2} v^{\beta}\right)-\cos \left(\frac{\pi}{2} v^{\beta}\right)\right] .
$$

Now, let us introduce the function $g(y ; \gamma)=\gamma y \sin (y)-\cos (y), y \in[0, \pi / 2]$, with $\gamma>0$. We have $g^{\prime}(y ; \gamma)=$ $(\gamma+1) \sin (y)+\gamma y \cos (y) \geq 0$, implying that $g(y ; \gamma)$ is increasing. Therefore for any $y \in[0, \pi / 2]$, we have $g(y ; \gamma) \in[g(0 ; \gamma), g(\pi / 2 ; \gamma)]=[-1, \gamma \pi / 2]$. Thus, thanks to the assumption makes on $\lambda$, we have

$$
\frac{\partial^{2} C_{\star}(u, v)}{\partial u \partial v}=1+\lambda g\left(\frac{\pi}{2} u^{\alpha} ; \alpha\right) g\left(\frac{\pi}{2} v^{\beta} ; \beta\right) \geq 1-|\lambda| \max \left(\frac{\alpha \pi}{2}, 1\right) \max \left(\frac{\beta \pi}{2}, 1\right) \geq 0 .
$$

The last required condition is shown. The proof of Proposition 1 is now complete.

To the best of our knowledge, Equation (3) defines a novel copula in the literature, and it is one of the few copulas that involves the cosine function. We call it PC copula, meaning "power-cosine" copula. The rest of the study is devoted to several theoretical and practical aspects of the PC copula.

\subsection{Immediate properties}

It is worth noting that for $\lambda=0$, we have $C_{\star}(u, v)=u v$, which corresponds to the independent copula. Based on the proof of Proposition 1, the PC copula density is obtained as

$$
c_{\star}(u, v)=\frac{\partial^{2} C_{\star}(u, v)}{\partial u \partial v}=1+\lambda\left[\alpha \frac{\pi}{2} u^{\alpha} \sin \left(\frac{\pi}{2} u^{\alpha}\right)-\cos \left(\frac{\pi}{2} u^{\alpha}\right)\right]\left[\beta \frac{\pi}{2} v^{\beta} \sin \left(\frac{\pi}{2} v^{\beta}\right)-\cos \left(\frac{\pi}{2} v^{\beta}\right)\right] .
$$

We can notice that this function is of relative complexity, involving several trigonometric terms. To understand this complexity and the most basic effect of the parameters, let us calculate this copula density at the boundaries; we have $c_{\star}(0,0)=1+\lambda, c_{\star}(1,1)=1+\lambda \alpha \beta \pi^{2} / 4, c_{\star}(0,1)=1-\lambda \beta \pi / 2$, and $c_{\star}(1,0)=1-\lambda \alpha \pi / 2$. It is also clear that all the parameters impact on the possible shapes of $c_{\star}(u, v)$. 
For $\alpha \neq \beta$, the equality $C_{\star}(u, v)=C_{\star}(v, u)$ is not satisfied for all $(u, v) \in[0,1]^{2}$; the PC copula is asymmetric. It is symmetric for $\alpha=\beta$. For $\lambda \in[0,1]$, we have $C_{\star}(u, v) \geq u v$, the positive quadrant dependence (PQD) property is satisfied. In addition, for $\lambda \in[-1,0]$, we have $C_{\star}(u, v) \leq u v$, the negative quadrant dependence (NQD) property is satisfied. As an additional property, like any copula, the PC copula satisfies the Fréchet-Hoeffding inequalities: for any $(u, v) \in[0,1]^{2}$, we have $\max (u+v-1,0) \leq C_{\star}(u, v) \leq \min (u, v)$. The reflected PC copula is specified by $\widehat{C}_{\star}(u, v)=u+v-1+C_{\star}(1-u, 1-v)$. In an expanded form, we get

$$
\widehat{C}_{\star}(u, v)=u v+\lambda(1-u)(1-v) \cos \left(\frac{\pi}{2}(1-u)^{\alpha}\right) \cos \left(\frac{\pi}{2}(1-v)^{\beta}\right) .
$$

Since $\widehat{C}_{\star}(u, v)=C_{\star}(u, v)$ is not satisfied for all $(u, v) \in[0,1]^{2}$, the PC copula is not radially symmetric. Also, to our knowledge, the copula $\widehat{C}_{\star}(u, v)$ is also a power-cosine-type copula that is not listed in the literature.

As an immediate measure of dependence, the medial correlation coefficient is obtained as

$$
M=4 C_{\star}\left(\frac{1}{2}, \frac{1}{2}\right)-1=\lambda \cos \left(\frac{\pi}{2^{\alpha+1}}\right) \cos \left(\frac{\pi}{2^{\beta+1}}\right) .
$$

We can see that the sign of $M$ is driven by the sign of $\lambda$, and that it tends to $\lambda$ when $\alpha$ or $\beta$ tend to $+\infty$, which itself tends to 1 . Hence, all the parameters have an effect on this measure. For further interpretations of the medial correlation coefficient, the reader is directed to [19].

\subsection{Illustrations}

We complete the above part with some graphics, to visualize the shapes of the main functions. More precisely, we provide the 3D and contour plots of the PC copula defined by Equation (3), along with the PC copula density in Equation (4). As an illustration, we chose the following sets of parameters at random: Set I: $(\alpha=1 / 2, \beta=2, \lambda=1 /(2 \pi))$, Set II: $(\alpha=2 / \pi, \beta=2 / \pi, \lambda=-1)$ and Set III: $(\alpha=1, \beta=1, \lambda=2 / 5)$. Figures 1,2 and 3 depict the PC copula for Sets I, II and III, respectively, while Figures 4, 5 and 6 depict the PC copula density for Sets I, II and III, respectively.
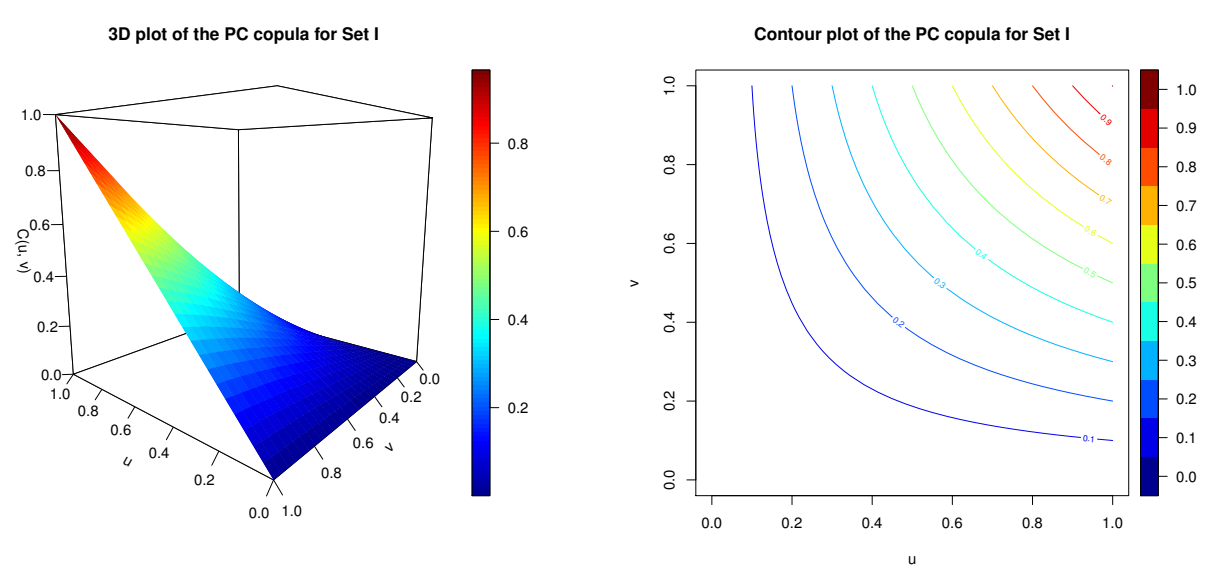

Figure 1. 3D and contour plots of the PC copula for Set I. 

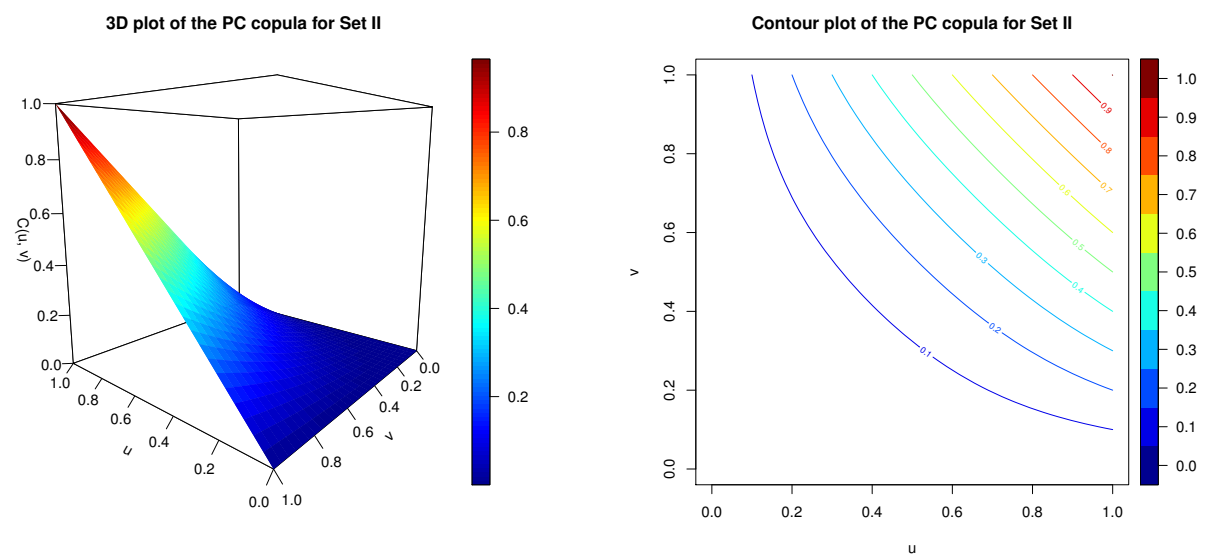

Figure 2. 3D and contour plots of the PC copula for Set II.
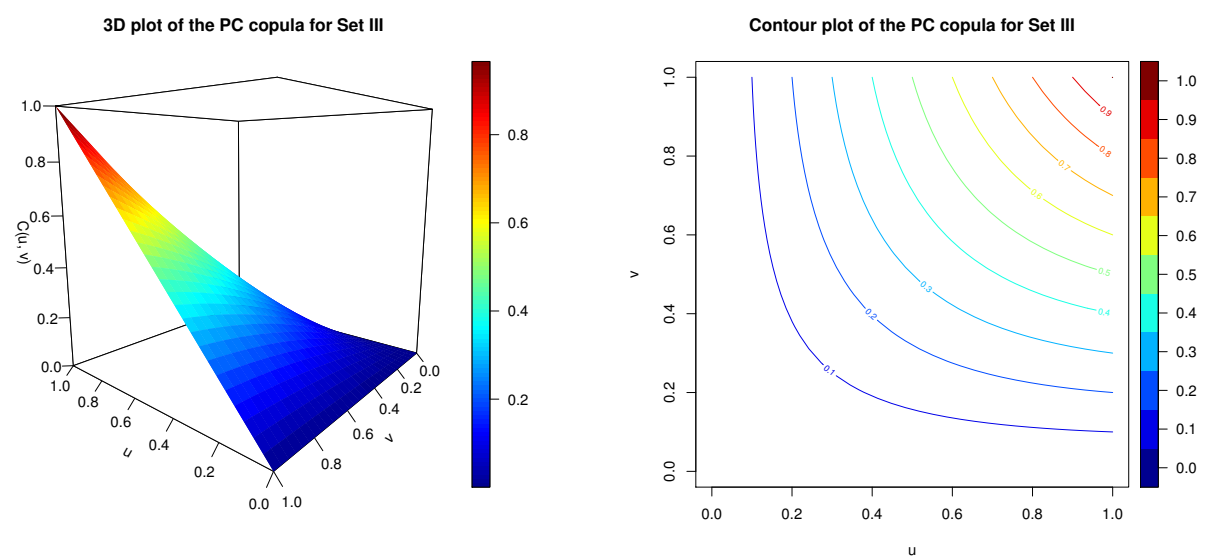

Figure 3. 3D and contour plots of the PC copula for Set III.
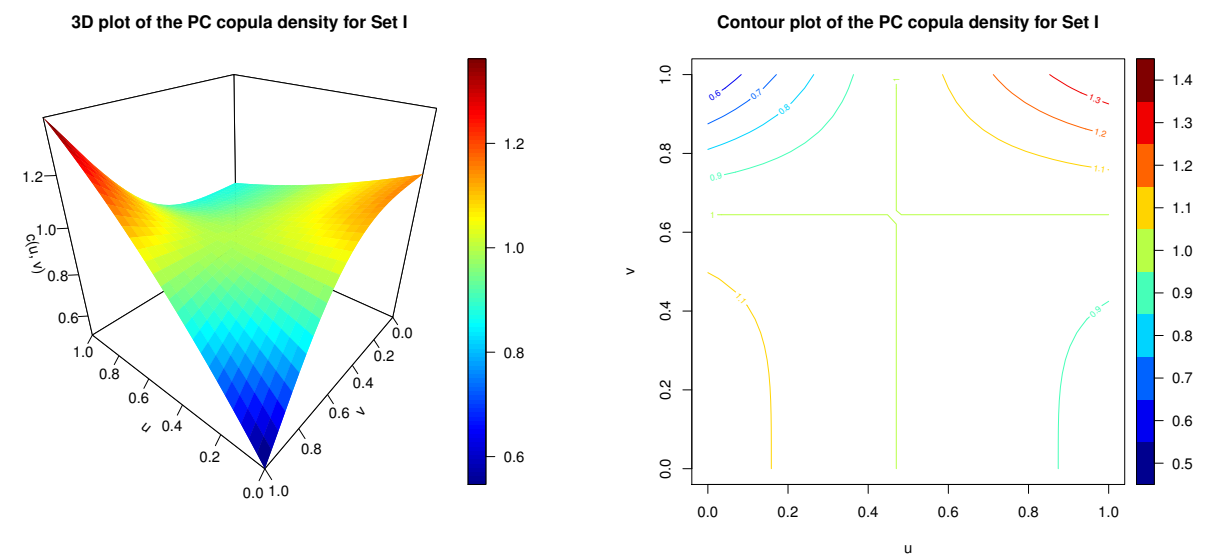

Figure 4. 3D and contour plots of the PC copula density for Set I. 

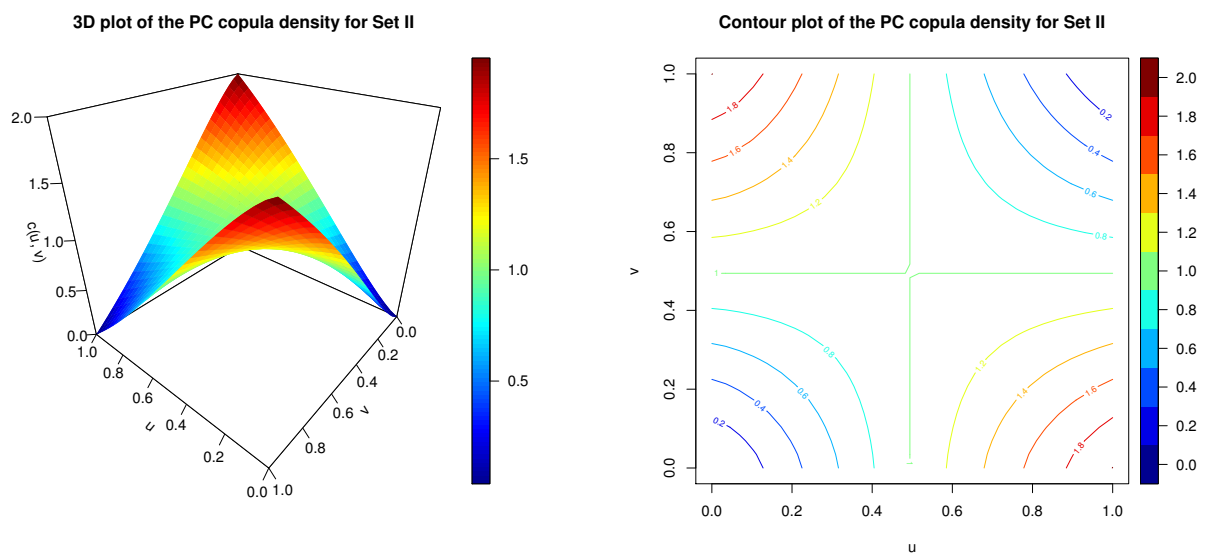

Figure 5. 3D and contour plots of the PC copula density for Set II.
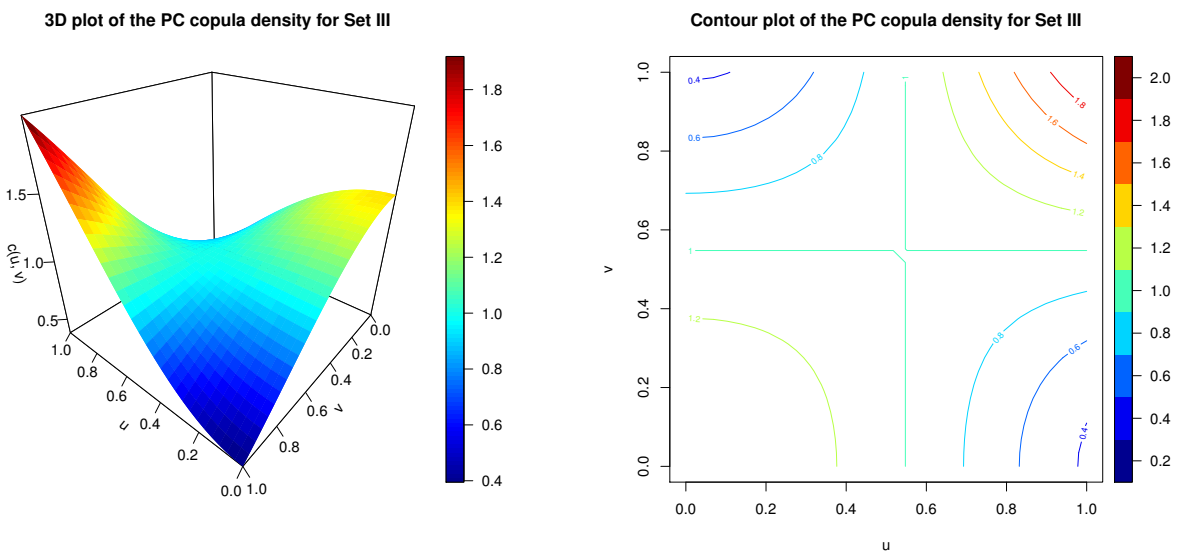

Figure 6. 3D and contour plots of the PC copula density for Set III.

From Figures 1, 2 and 3, we see that the PC copula has a triangle-like shape that wraps around itself based on the parameter values. Figures 4, 5 and 6 show that the PC copula density is highly adjustable, with a wide range of form behaviors, mostly in the boundary neighborhoods.

\subsection{A new bivariate normal distribution}

As an immediate application, the PC copula can be used to introduce new generalized bivariate distributions. For example, the PC normal (PCN) distribution can be created by using the normal distribution as a parental distribution. It is described below:

Let $\mu \in \mathbb{R}, \sigma>0$ and

$$
\Phi(x ; \mu, \sigma)=\int_{-\infty}^{x} \phi(t ; \mu, \sigma) d t, \quad \phi(x ; \mu, \sigma)=\frac{1}{\sigma \sqrt{2 \pi}} \exp \left(-\frac{(x-\mu)^{2}}{2 \sigma^{2}}\right), \quad x \in \mathbb{R} .
$$

Then, based on Equation (3), the following CDF defines the PCN distribution:

$$
F(x, y)=C_{\star}\left(\Phi\left(x ; \mu_{1}, \sigma_{1}\right), \Phi\left(y ; \mu_{2}, \sigma_{2}\right)\right), \quad(x, y) \in \mathbb{R}^{2},
$$

with $\mu_{1}, \mu_{2} \in \mathbb{R}$ and $\sigma_{1}, \sigma_{2}>0$. That is, in an expanded form, for $(x, y) \in \mathbb{R}^{2}$,

$$
F(x, y)=\Phi\left(x ; \mu_{1}, \sigma_{1}\right) \Phi\left(y ; \mu_{2}, \sigma_{2}\right)\left[1+\lambda \cos \left(\frac{\pi}{2} \Phi\left(x ; \mu_{1}, \sigma_{1}\right)^{\alpha}\right) \cos \left(\frac{\pi}{2} \Phi\left(y ; \mu_{2}, \sigma_{2}\right)^{\beta}\right)\right] .
$$


The presence of power and cosine terms is one of the main features of this bivariate distribution. Based on Equation (4), the corresponding probability density function (PDF) is obtained as

$$
f(x, y)=\phi\left(x ; \mu_{1}, \sigma_{1}\right) \phi\left(y ; \mu_{2}, \sigma_{2}\right) c_{\star}\left(\Phi\left(x ; \mu_{1}, \sigma_{1}\right), \Phi\left(y ; \mu_{2}, \sigma_{2}\right)\right), \quad(x, y) \in \mathbb{R}^{2} .
$$

Hence, for $(x, y) \in \mathbb{R}^{2}$, we have

$$
\begin{aligned}
f(x, y)= & \phi\left(x ; \mu_{1}, \sigma_{1}\right) \phi\left(y ; \mu_{2}, \sigma_{2}\right)\left\{1+\lambda\left[\alpha \frac{\pi}{2} \Phi\left(x ; \mu_{1}, \sigma_{1}\right)^{\alpha} \sin \left(\frac{\pi}{2} \Phi\left(x ; \mu_{1}, \sigma_{1}\right)^{\alpha}\right)-\cos \left(\frac{\pi}{2} \Phi\left(x ; \mu_{1}, \sigma_{1}\right)^{\alpha}\right)\right]\right. \\
& \left.\times\left[\beta \frac{\pi}{2} \phi\left(y ; \mu_{2}, \sigma_{2}\right)^{\beta} \sin \left(\frac{\pi}{2} \phi\left(y ; \mu_{2}, \sigma_{2}\right)^{\beta}\right)-\cos \left(\frac{\pi}{2} \phi\left(y ; \mu_{2}, \sigma_{2}\right)^{\beta}\right)\right]\right\} .
\end{aligned}
$$

The standard PCN (SPCN) distribution is defined as the PCN distribution with $\mu_{1}=\mu_{2}=0$, and $\sigma_{1}=$ $\sigma_{2}=1$. Due to its bivariate complexity, it is difficult to study its analytical behavior. For this reason, we give a direct view of it by providing some 3D and contour plots of the CDF of the SPCN distribution for Sets I, II and III in Figures 7, 8 and 9, respectively, and the associated 3D and contour plots of the PDF for Sets I, II and III in Figures 10, 11 and 12, respectively.
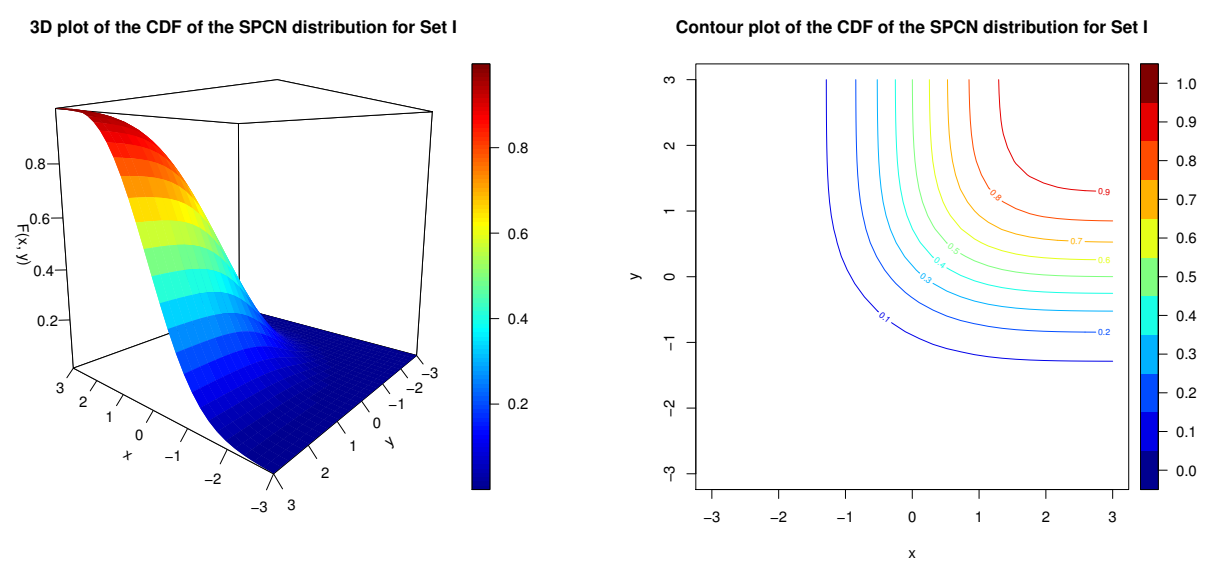

Figure 7. 3D and contour plots of the CDF of the SPCN distribution for Set I.
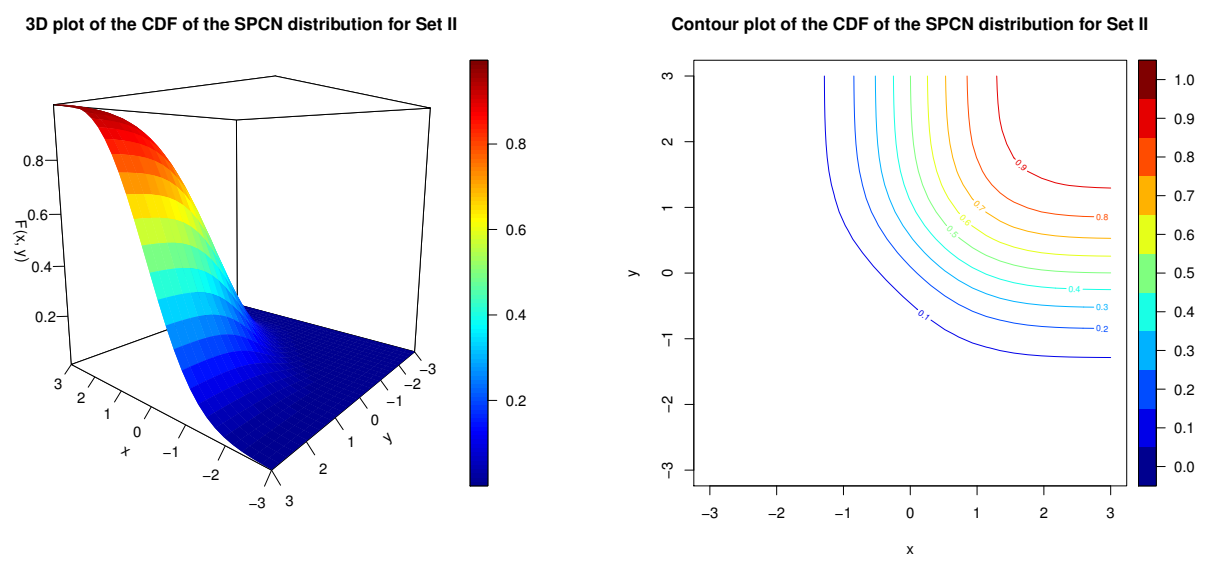

Figure 8. 3D and contour plots of the CDF of the SPCN distribution for Set II. 
3D plot of the CDF of the SPCN distribution for Set III

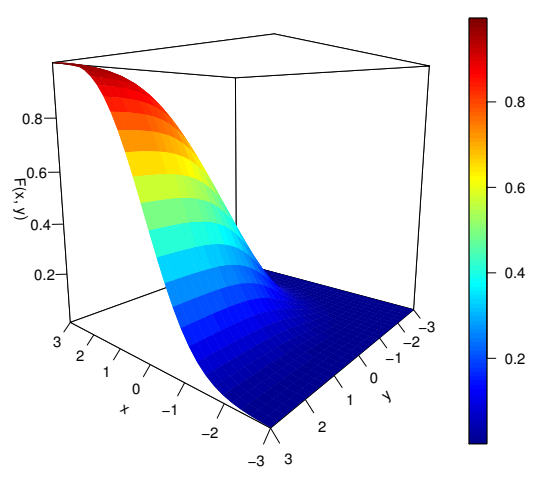

Contour plot of the CDF of the SPCN distribution for Set III

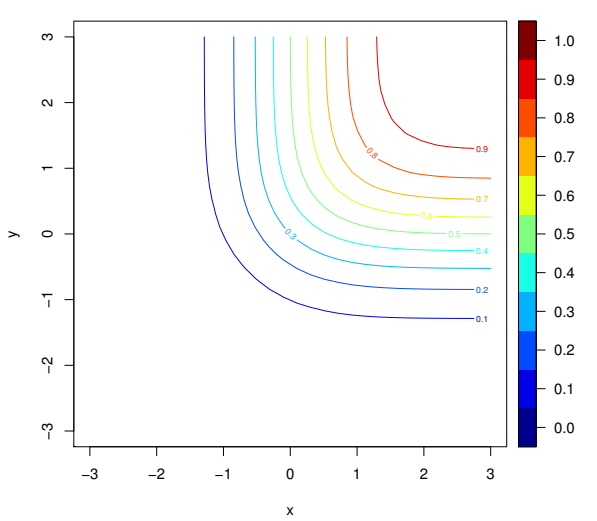

Figure 9. 3D and contour plots of the CDF of the SPCN distribution for Set III.
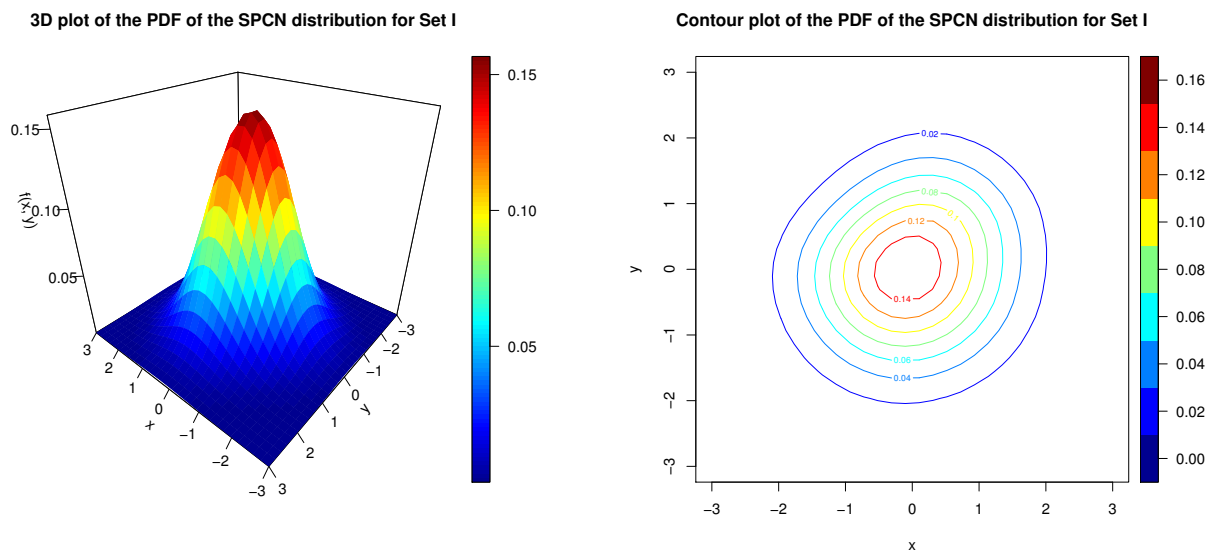

Figure 10. 3D and contour plots of the PDF of the SPCN distribution for Set I.
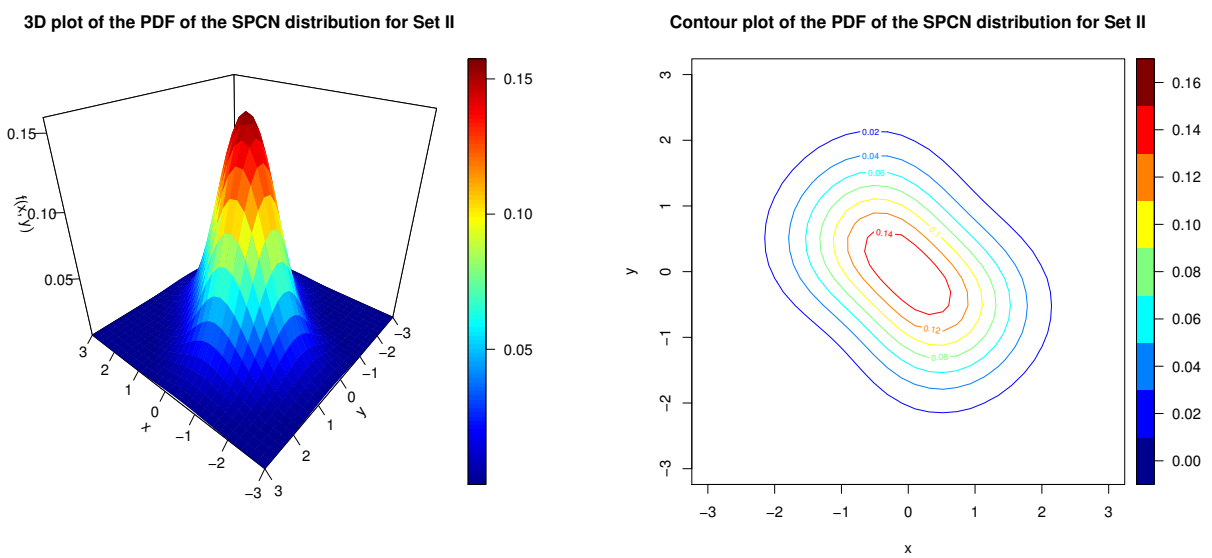

Figure 11. 3D and contour plots of the PDF of the SPCN distribution for Set II. 

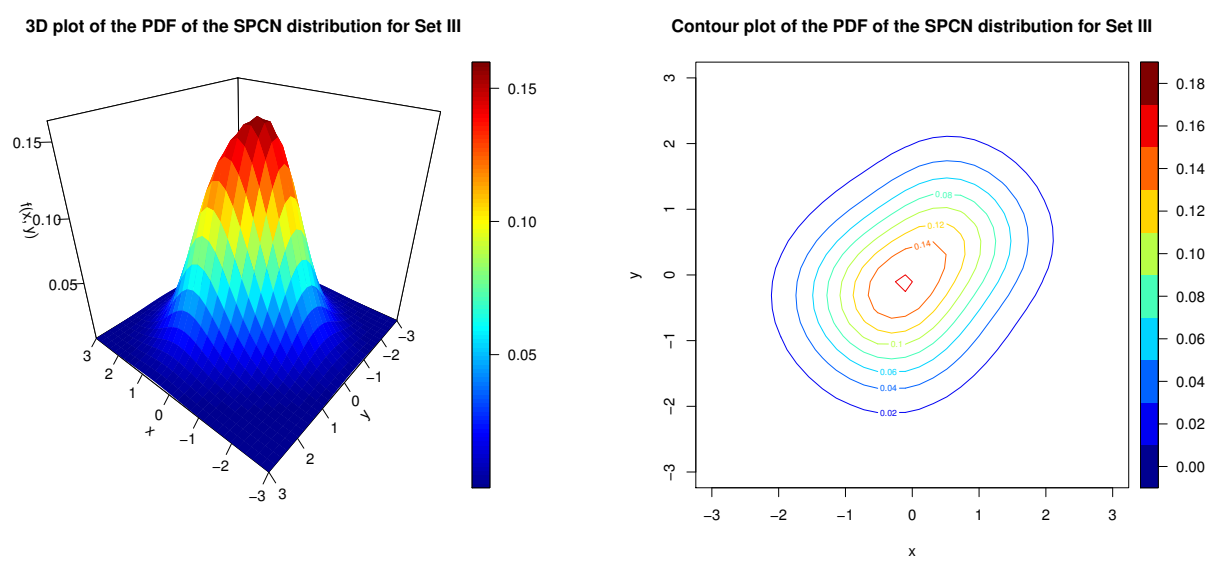

Figure 12. 3D and contour plots of the PDF of the SPCN distribution for Set III.

From Figures 10, 11 and 12, we see that, depending on the value of the parameters, the copula density deforms into a bell shape. This is especially apparent in the contour plots.

Like any bivariate normal distribution, the PCN distribution can be used for a variety of statistical purposes, such as data analysis. A classical scenario is described below. Consider $n$ pairs of data $\left(x_{1}, y_{1}\right), \ldots,\left(x_{n}, y_{n}\right)$ which are assumed to be observed from a bivariate random vector $(X, Y)$ with the PCN distribution. We can estimate the supposed unknown parameters of the PCN distribution via the maximum likelihood procedure; $\mu_{1}, \mu_{2}, \sigma_{1}, \sigma_{2}$ and $\lambda$ can be estimated by $\tilde{\mu}_{1}, \tilde{\mu}_{2}, \tilde{\sigma}_{1}, \tilde{\sigma}_{2}$ and $\tilde{\lambda}$, where

$$
\left(\tilde{\mu}_{1}, \tilde{\mu}_{2}, \tilde{\sigma}_{1}, \tilde{\sigma}_{2}, \tilde{\lambda}\right)=\operatorname{argmax}_{\left(\mu_{1}, \mu_{2}, \sigma_{1}, \sigma_{2}, \lambda\right)} \sum_{i=1}^{n} \log \left[f\left(x_{i}, y_{i}\right)\right] .
$$

As a result, we can estimate $f(x, y)$ by substituting the unknown parameters by their respective estimates. As a result, we construct a functional estimate $\tilde{f}(x, y)$ of $f(x, y)$, which is required for a precise analysis of the data. We refer to [20] for the details on the maximum likelihood procedure. Alternative estimation procedures can be found in [21]. Other directions of applied work include clustering and regression modeling. Such in-depth applications necessitate additional research, which we will postpone for now.

\subsection{Extensions of the PC copula}

In this subsection, we briefly discuss several possible extensions of the PC copula.

Copula distortions are a simple approach to generate new copulas from a parental copula. The possibility of improving the fit of an initial copula is one of the benefits of such distortions. Based on the PC copula and a distortion function denoted by $T(x)$, the generalized form of the distorted PC copula is

$$
\check{C}_{\star}(u, v)=T\left(C_{\star}\left(T^{-1}(u), T^{-1}(v)\right)\right), \quad(u, v) \in[0,1]^{2} .
$$

That is, in an expanded form, for $(u, v) \in[0,1]^{2}$,

$$
\check{C}_{\star}(u, v)=T\left(T^{-1}(u) T^{-1}(v)\left[1+\lambda \cos \left(\frac{\pi}{2}\left[T^{-1}(u)\right]^{\alpha}\right) \cos \left(\frac{\pi}{2}\left[T^{-1}(v)\right]^{\beta}\right)\right]\right) .
$$

Basically, $T(x)$ is an increasing function such that $T(0)=0$ and $T(1)=1$, with supplementary technical assumptions that must be chosen to guarantee that $\breve{C}_{\star}(u, v)$ is also a valid copula. The reader can be directed to [22] for more information on such a distorted function.

We conclude with a corollary concerning a natural multivariate expansion of the PC copula. 
Corollary 1. Let $d$ be a strictly positive integer, $\alpha_{1}, \alpha_{2}, \ldots, \alpha_{d}>0$, and $\lambda \in\left[-c^{-1}, c^{-1}\right]$, where $c=$ $\prod_{i=1}^{d} \max \left(\alpha_{i} \pi / 2,1\right)$. Then the following multivariate function is a copula:

$$
C_{\star}\left(u_{1}, u_{2}, \ldots, u_{d}\right)=\left[\prod_{i=1}^{n} u_{i}\right]\left[1+\lambda \prod_{i=1}^{n} \cos \left(\frac{\pi}{2} u_{i}^{\alpha_{i}}\right)\right], \quad\left(u_{1}, u_{2}, \ldots, u_{d}\right) \in[0,1]^{d} .
$$

The proof can be done in the same way as Proposition 1 was proved.

Thus, the multivariate PC (MPC) copula is the multivariate variation of the PC copula. It can be used to develop multivariate statistical models and generalized multivariate distributions.

\section{On the simple PC copula}

We now propose to look into the most basic situation of the PC copula, which involves cosine terms with no power parameters.

\subsection{Presentation}

When $\alpha=\beta=1$, the PC copula in Equation (3) is well-defined with $\lambda \in\left[-4 / \pi^{2}, 4 / \pi^{2}\right]$, and it is reduced to the following simple form:

$$
C_{\star}(u, v)=u v\left[1+\lambda \cos \left(\frac{\pi}{2} u\right) \cos \left(\frac{\pi}{2} v\right)\right], \quad(u, v) \in[0,1]^{2} .
$$

To the best of our knowledge, it is not listed in the literature. In this special case, we call it the simple PC (SPC) copula. We mention that it covers the independent case, is symmetric, satisfies the PQD property for $\lambda \in\left[0,4 / \pi^{2}\right]$ and the NQD property for $\lambda \in\left[-4 / \pi^{2}, 0\right]$, the Fréchet-Hoeffding inequalities, and is not radially symmetric. The corresponding SPC copula density is given as

$$
c_{\star}(u, v)=1+\lambda\left[\frac{\pi}{2} u \sin \left(\frac{\pi}{2} u\right)-\cos \left(\frac{\pi}{2} u\right)\right]\left[\frac{\pi}{2} v \sin \left(\frac{\pi}{2} v\right)-\cos \left(\frac{\pi}{2} v\right)\right]
$$

and the medial correlation coefficient is reduced to $M=\lambda / 2$. The reflected SPC copula is specified by

$$
\widehat{C}_{\star}(u, v)=u v+\lambda(1-u)(1-v) \sin \left(\frac{\pi}{2} u\right) \sin \left(\frac{\pi}{2} v\right) .
$$

It is a polynomial-sine copula that looks a lot like the SPS copula, but it is not mentioned in the literature as far as we know.

Finally, the SPC copula and copula density were plotted in Figures 3 and 6, respectively, for $\lambda=2 / 5$.

\subsection{Ordering properties}

The following result shows some ordering properties between the FGM, SPS and SPC copulas.

Proposition 2. Let $C_{\circ}(u, v ; \lambda)=C_{\circ}(u, v)$ be the FGM copula as given in Equation (1), $C_{\triangle}(u, v ; \lambda)=C_{\triangle}(u, v)$ be the SPS copula as given in Equation (2), and $C_{\star}(u, v ; \lambda)=C_{\star}(u, v)$ be the SPC copula as given in Equation (5). Then the following results hold.

- The FGM and SPC copulas can be compared as follows: for $\lambda \in\left[0,4 / \pi^{2}\right]$, we have

$$
C_{\circ}(u, v) \leq C_{\star}(u, v) \leq C_{\circ}\left(u, v ; \lambda \frac{\pi^{2}}{4}\right) .
$$

- The SPS and SPC copulas can be compared as follows: for $\lambda \in\left[0,4 / \pi^{2}\right]$, we have

$$
C_{\star}\left(u, v ; \lambda \frac{4}{\pi^{2}}\right) \leq C_{\triangle}(u, v) \leq C_{\star}(u, v) .
$$

For each item, the reversed double inequalities hold for $\lambda \in\left[-4 / \pi^{2}, 0\right]$. 
Proof. First, a trigonometric formula gives $\cos ((\pi / 2) u)=\sin ((\pi / 2)(1-u))$, so we can write $C_{\star}(u, v)$ as

$$
C_{\star}(u, v)=u v\left[1+\lambda \sin \left(\frac{\pi}{2}(1-u)\right) \sin \left(\frac{\pi}{2}(1-v)\right)\right]
$$

Let us now show the two itemized results, in turn.

- The following double inequality is satisfied: $(2 / \pi) y \leq \sin (y) \leq y$ for $y \in[0, \pi / 2]$. As a direct application, we have

$$
(1-u)(1-v) \leq \sin \left(\frac{\pi}{2}(1-u)\right) \sin \left(\frac{\pi}{2}(1-v)\right) \leq \frac{\pi^{2}}{4}(1-u)(1-v) .
$$

By taking into account Equations (6) and the definition of the FGM copula with $\lambda \in\left[0,4 / \pi^{2}\right]$, the stated double copula inequality is obtained. For $\lambda \in\left[-4 / \pi^{2}, 0\right]$, the reversed holds.

- By a remark in [16], we can write $C_{\triangle}(u, v ; \lambda)$ as

$$
C_{\triangle}(u, v ; \lambda)=u v+\lambda \frac{4}{\pi^{2}} \sin \left(\frac{\pi}{2} u\right) \sin \left(\frac{\pi}{2} v\right) \sin \left(\frac{\pi}{2}(1-u)\right) \sin \left(\frac{\pi}{2}(1-v)\right) .
$$

Owing to the following double inequality: $(2 / \pi) y \leq \sin (y) \leq y$ for $y \in[0, \pi / 2]$, we get

$$
\begin{aligned}
u v \sin \left(\frac{\pi}{2}(1-u)\right) \sin \left(\frac{\pi}{2}(1-v)\right) & \leq \sin \left(\frac{\pi}{2} u\right) \sin \left(\frac{\pi}{2} v\right) \sin \left(\frac{\pi}{2}(1-u)\right) \sin \left(\frac{\pi}{2}(1-v)\right) \\
& \leq \frac{\pi^{2}}{4} u v \sin \left(\frac{\pi}{2}(1-u)\right) \sin \left(\frac{\pi}{2}(1-v)\right) .
\end{aligned}
$$

By multiplying this double inequality by $\lambda\left(4 / \pi^{2}\right)$ with $\lambda \in\left[0,4 / \pi^{2}\right]$, and using the expressions in Equations (6) and (7), the indicated double copula inequality is obtained. For $\lambda \in\left[-4 / \pi^{2}, 0\right]$, the reversed holds.

This concludes the proof of Proposition 2.

Proposition 2 demonstrates that, under certain conditions, the FGM, SPS, and SPC copulas have comprehensive ordering properties; the SPC copula may be uniformly superior or inferior to the FGM copula, and vice versa, depending on the values of $\lambda$. The case of $|\lambda|>4 / \pi^{2}$ is excluded from the simple copula ordering since the SPC copula is not well defined for such values of $\lambda$, contrary to the FGM and SPS copulas. Consequently, the FGM, SPS and SPC copulas can achieve different objectives in terms of copula modelling.

\subsection{Family membership}

In this subsection, we show that the SPC copula is a member of the copula family introduced by [15]. This is formulated in the following proposition.

Proposition 3. Under the following configuration, the SPC copula belongs to the semi-parametric family of bivariate copulas introduced in [15]:

$$
\theta=\lambda \frac{\pi^{2}}{4}, \quad \phi(u)=\frac{2}{\pi} u \cos \left(\frac{\pi}{2} u\right)
$$

Proof. Based on Equation (5), we can write the SPC copula as

$$
C_{\star}(u, v)=u v\left[1+\lambda \cos \left(\frac{\pi}{2} u\right) \cos \left(\frac{\pi}{2} v\right)\right]=u v+\theta \phi(u) \phi(v),
$$

with $\theta=\lambda \pi^{2} / 4 \in[-1,1]$ and $\phi(u)=(2 / \pi) u \cos ((\pi / 2) u)$. It is clear that $\phi(0)=\phi(1)=0$. To end the proof, we must show that $\phi(u)$ satisfies the Lipschitz condition: $|\phi(x)-\phi(y)| \leq|x-y|$ for any $(x, y) \in[0,1]^{2}$. In particular, this condition is satisfied if $\left|\phi^{\prime}(u)\right| \leq 1$ for all $u \in[0,1]$. Here, we have $\phi^{\prime}(u)=(2 / \pi) \cos ((\pi / 2) u)-$ $u \sin ((\pi / 2) u)$ and $\phi^{\prime \prime}(u)=-2 \sin ((\pi / 2) u)-(\pi / 2) u \cos ((\pi / 2) u)$. So $\phi^{\prime \prime}(u) \leq 0$ for $u \in[0,1]$, implying that $\phi^{\prime}(u)$ is decreasing: we have $\phi^{\prime}(u) \in\left[\phi^{\prime}(1), \phi^{\prime}(0)\right]=[-1,2 / \pi] \subseteq[-1,1]$. Therefore $\left|\phi^{\prime}(u)\right| \leq 1$ and the Lipschitz condition is satisfied. This concludes the proof of Proposition 3. 
Proposition 3 implies that all the results in [15] can be applied to the SPC copula under the mentioned configuration on $\theta$ and $\phi(u)$. In particular, thanks to [15, Theorem 3], we can notice that $\phi(u) / u$ and $\phi(u) /(u-$ 1 ) are decreasing for $u \in[0,1]$, implying that the SPC copula has the left and right tail properties, respectively. Furthermore, because $\phi^{\prime \prime}(u) \leq 0$ for any $u \in[0,1], \phi(u)$ is concave, we can claim that the SPC copula has the stochastically increasing property.

\subsection{Dependent properties}

Measures of dependence are commonly employed to describe a complicated dependency structure in a bivariate scenario. We have already briefly presented the medial correlation coefficient of the SPC copula. In this subsection, the dependence of the SPC is examined through the normalized volume, Kendall and Spearman measures. When they are associated with a copula $C(u, v)$, they are defined by

$$
v=12 \int_{0}^{1} \int_{0}^{1}|C(u, v)-u v| d u d v, \quad \tau=1-4 \int_{0}^{1} \int_{0}^{1} \frac{\partial C(u, v)}{\partial u} \frac{\partial C(u, v)}{\partial v} d u d v
$$

and

$$
\rho=12 \int_{0}^{1} \int_{0}^{1} C(u, v) d u d v-3
$$

respectively. For further information, alternate formulations, and details on these measurements, see [23].

These measures are reflected in the next result when the SPC copula is taken into account.

Proposition 4. The normalized volume, Kendall and Spearman measures of dependence related to the SPC copula are given by

$$
v=192 \frac{(\pi-2)^{2}}{\pi^{6}}|\theta|, \quad \tau=128 \frac{(\pi-2)^{2}}{\pi^{6}} \theta, \quad \rho=192 \frac{(\pi-2)^{2}}{\pi^{6}} \theta,
$$

respectively, with $\theta=\lambda \pi^{2} / 4 \in[-1,1]$.

Proof. By virtue of Proposition 3, we can apply [15, Proposition 1]. The following direct expressions are obtained:

$$
v=12|\theta|\left(\int_{0}^{1}|\phi(u)| d u\right)^{2}, \quad \tau=8 \theta\left(\int_{0}^{1} \phi(u) d u\right)^{2}, \quad \rho=\frac{3}{2} \tau,
$$

with $\theta=\lambda \pi^{2} / 4 \in[-1,1]$ and $\phi(u)=(2 / \pi) u \cos ((\pi / 2) u)$. Since $\phi(u) \geq 0$ for any $u \in[0,1]$, an integration by part gives

$$
\int_{0}^{1}|\phi(u)| d u=\int_{0}^{1} \phi(u) d u=\frac{2}{\pi} \int_{0}^{1} u \cos ((\pi / 2) u) d u=\frac{4(\pi-2)}{\pi^{3}} .
$$

Therefore

$$
v=12|\theta|\left(\frac{4(\pi-2)}{\pi^{3}}\right)^{2}=192 \frac{(\pi-2)^{2}}{\pi^{6}}|\theta|, \quad \tau=8 \theta\left(\frac{4(\pi-2)}{\pi^{3}}\right)^{2}=128 \frac{(\pi-2)^{2}}{\pi^{6}} \theta
$$

and

$$
\rho=\frac{3}{2} 128 \frac{(\pi-2)^{2}}{\pi^{6}} \theta=192 \frac{(\pi-2)^{2}}{\pi^{6}} \theta
$$

This ends the proof of Proposition 4.

It can be noted that $128(\pi-2)^{2} / \pi^{6} \approx 0.1735134$, and $192(\pi-2)^{2} / \pi^{6} \approx 0.2602701$, giving the following approximative dependence domains: $v \in[0,0.26], \tau \in[-0.18,0.18]$ and $\rho \in[-0.26,0.26]$. These domains are slightly smaller than those reached by the FGM and SPS copulas. Thus, the SPC copula must be used for the modelling of moderate dependences.

\subsection{Tail dependence}

The level of dependency in the upper-right quadrant tail and lower-left quadrant tail of a bivariate distribution is referred to as tail dependence. The notions below are considered. 
- Let us define $\lambda_{U}$ as

$$
\lambda_{U}=\lim _{u \rightarrow 1} \frac{1-2 u+C(u, u)}{1-u} .
$$

If $\lambda_{U}$ exists, then $C(u, v)$ has an upper tail dependence if $\lambda_{U} \in(0,1]$, and an upper tail independence if $\lambda_{U}=0$.

- Let us define $\lambda_{L}$ as

$$
\lambda_{L}=\lim _{u \rightarrow 0} \frac{C(u, u)}{u}
$$

If $\lambda_{L}$ exists, then $C(u, v)$ has a lower tail dependence if $\lambda_{L} \in(0,1]$, and a lower tail independence if $\lambda_{L}=0$.

These two measurements are heavily used in extreme value theory. [24] has more information on the tail dependence. In the next result, we look into them for the SPC copula.

Proposition 5. The SPC copula has a total tail independence; we have $\lambda_{U}=\lambda_{L}=0$.

Proof. Owing to Equation (5), when $u \rightarrow 1$, we get

$$
\frac{1-2 u+C_{\star}(u, u)}{1-u}=1-u+\lambda \frac{u^{2}[\cos ((\pi / 2) u)]^{2}}{1-u} \sim(1-u)\left(1+\lambda \frac{\pi^{2}}{4}\right) \rightarrow 0 .
$$

Hence $\lambda_{U}=0$.

Moreover, when $u \rightarrow 0$, we obtain

$$
\frac{C_{\star}(u, u)}{u}=u\left\{1+\lambda\left[\cos \left(\frac{\pi}{2} u\right)\right]^{2}\right\} \sim u(1+\lambda) \rightarrow 0 .
$$

We deduce that $\lambda_{L}=0$. The proof of Proposition 5 is now complete.

\section{Summary with discussion}

In this article, we have developed a new bivariate trigonometric copula, called the power-cosine copula. We have gone over it in depth, examining its copula density, reflected copula and its immediate properties. Also, it was shown how it can be used to create a new bivariate normal distribution. The most basic example of the power-cosine copula was then investigated. Some relationships with the Farlie-Gumbel-Morgensten and simple polynomial-sine copulas are highlighted, as well as its membership in a well-known family of copulas, determination of its main dependence domains, and tail dependence property. The newly constructed copula is thought to be useful in modeling bivariate phenomena with real data sets by practitioners due to its innovative trigonometric structure and complete features. In addition to such concrete applications, a possible sequel to this work is the study of the reflected power-cosine copula, which remains another option never explored before.

Acknowledgments: The author wants to express his gratitude to the two anonymous reviewers for their suggestions and comments.

Conflicts of Interest: "The author declares no conflict of interest."

\section{References}

[1] Sklar, M. (1959). Fonctions de répartition àn dimensions et leurs marges, Publ. L'Institut Stat. L'Université Paris, 8, 229-231.

[2] Georges, P., Lamy, A. G., Nicolas, E., Quibel, G., \& Roncalli, T. (2001). Multivariate survival modelling: a unified approach with copulas. Available at SSRN 1032559.

[3] Frees, E. W., \& Valdez, E. A. (1998). Understanding relationships using copulas. North American Actuarial Journal, 2(1), $1-25$.

[4] Kazianka, H., \& Pilz, J. (2010). Copula-based geostatistical modeling of continuous and discrete data including covariates. Stochastic Environmental Research and Risk Assessment, 24(5), 661-673.

[5] Zhang, Q., Chen, Y. D., Chen, X., \& Li, J. (2011). Copula-based analysis of hydrological extremes and implications of hydrological behaviors in the Pearl River basin, China. Journal of Hydrologic Engineering, 16(7), 598-607. 
[6] Kilgore, R. T., \& Thompson, D. B. (2011). Estimating joint flow probabilities at stream confluences by using copulas. Transportation Research Record, 2262(1), 200-206.

[7] Shiau, J. T., Wang, H. Y., \& Tsai, C. T. (2006). Bivariate frequency analysis of floods using copulas. JAWRA Journal of the American Water Resources Association, 42(6), 1549-1564.

[8] Trivedi, P. K., \& Zimmer, D. M. (2007). Copula Modeling: an Introduction for Practitioners. Now Publishers Inc.

[9] Nelsen, R. B. (2007). An Introduction to Copulas. Springer Science \& Business Media.

[10] Yong-quan, D. (2008). Generation and prolongation of fgm copula. Chinese Journal of Engineering Mathematics, 25, 1137-1141.

[11] Fischer, M., \& Klein, I. (2007). Constructing generalized FGM copulas by means of certain univariate distributions. Metrika, 65(2), 243-260.

[12] Bekrizadeh, H., Parham, G. A., \& Zadkarami, M. R. (2015). An asymmetric generalized FGM copula and its properties. Pakistan Journal of Statistics, 31(1), 95-106.

[13] Bekrizadeh, H., \& Jamshidi, B. (2017). A new class of bivariate copulas: dependence measures and properties. Metron, 75(1), 31-50.

[14] Bekrizadeh, H., Parham, G. A., \& Zadkarmi, M. R. (2012). The new generalization of Farlie-Gumbel-Morgenstern copulas. Applied Mathematical Sciences, 6(71), 3527-3533.

[15] Amblard, C., \& Girard, S. (2002). Symmetry and dependence properties within a semiparametric family of bivariate copulas. Journal of Nonparametric Statistics, 14(6), 715-727.

[16] Chesneau, C. (2021). A note on a simple polynomial-sine copula. Preprint.

[17] Durante, F. (2006). A new class of symmetric bivariate copulas. Nonparametric Statistics, 18(7-8), 499-510.

[18] Li, X., \& Fang, R. (2012). A new family of bivariate copulas generated by univariate distributions. Journal of Data Science, 10(1), 107-127.

[19] Domma, F. (2010). Some properties of the bivariate Burr type III distribution. Statistics, 44(2), $203-215$.

[20] Casella, G. \& Berger, R.L. (1990). Statistical Inference. Brooks/Cole Publishing Company: Bel Air, CA, USA.

[21] Abd Elaal, M. K., \& Jarwan, R. S. (2017). Inference of bivariate generalized exponential distribution based on copula functions. Applied Mathematical Sciences, 11(24), 1155-1186.

[22] Di Bernardino, E., \& Rullière, D. (2013). Distortions of multivariate distribution functions and associated level curves: Applications in multivariate risk theory. Insurance: Mathematics and Economics, 53(1), 190-205.

[23] Schweizer, B., \& Wolff, E. F. (1981). On nonparametric measures of dependence for random variables. Annals of Statistics, 9(4), 879-885.

[24] Coles, S., Heffernan, J., \& Tawn, J. (1999). Dependence measures for extreme value analyses. Extremes, 2(4), 339-365.

(C) 2021 by the authors; licensee PSRP, Lahore, Pakistan. This article is an open access article distributed under the terms and conditions of the Creative Commons Attribution (CC-BY) license (http://creativecommons.org/licenses/by/4.0/). 